\title{
Hybridisation of Evolutionary Programming and Machine Learning with $k$-Nearest Neighbor Estimation
}

\author{
Jingsong He, Zhenyu Yang, Xin Yao, Fellow, IEEE
}

\begin{abstract}
Evolutionary programming(EP) focus on the search step size which decides the ability of escaping local minima, however does not touch the issue of search in promising region. Estimation of Distribution Algorithms(EDAs) focus on where the promising region is, however have less consideration about behavior of each individual in solution search algorithms. Since the basic ideas of EP and EDAs are quite different, it is possible to make them reinforce each other. In this paper, we present a hybrid evolutionary framework to make use of both the ideas of EP and EDAs through introducing a mini estimation operator into EP's search cycle. Unlike previous EDAs that use probability density function(PDF), the estimation mechanism used in the proposed framework is the $k$-nearest neighbor estimation which can perform better with relative small amount of training samples. Our experimental results have shown that the incorporation of machine learning techniques, such as knearest neighbor estimation, can improve the performance of evolutionary optimisation algorithms for a large number of benchmark functions.
\end{abstract}

\section{INTRODUCTION}

Evolutionary programming (EP)[1] is one of the most important optimization methodology in the field of evolutionary computation, and has been applied with success to many numerical and combinatorial optimization problems[25]. Although there are many empirical and theoretical studies improved EP's performance in past years, e.g.,[6][7][8][9], the basic idea for optimization by EP is yet with two major steps: mutation and selection. The generate-and-test formulation of EP indicates that mutation is a key search operator which generates new solutions from the current ones. Fogel [3][10] and Bäck and Schwefel [11] have indicated that EP with self-adaptive mutation usually performs better than EP without self-adaptive mutation for the functions they tested. Continuous studies showed that long jumps such as Cauchy mutations[6] and Lévy mutations[7] can help individuals escaping local minima efficiently. However, EP's search strategies does not care about where is the promising region it should go, but to escape local minima.

Estimation of Distribution Algorithms (EDAs) are another approach method of population based search algorithms that generate new population from the estimated distribution based on current promising solutions. Early EDAs

The authors are with Nature Inspired Computation and Applications Laboratory, University of Science and Technology of China (USTC), Hefei, Anhui 230027, China. Jingsong He is also with the Department of Electronic Science and Technology at USTC. Xin Yao is also with CERCIA, School of Computer Science, University of Birmingham, Birmingham B15 2TT, UK. Email: \{hjss@ustc.edu.cn, zhyuyang@mail.ustc.edu.cn, X.Yao@cs.bham.ac.uk \}

This work is partially supported by the National Natural Science Foundation of China through Grant No.60573170 and Grant No. 60428202. for continuous optimization presumed that selected vector is a random sample from a single Gaussian distribution. Improved versions of EDAs turn to using Gaussian mixture distributions[12][13][14][15] by incorporating clustering techniques. Obviously, new populations generated based on a wrong estimation would be likely to be in the wrong search area, and vice versa. The performance of EDAs depends on whether the predictive accuracy of estimator is higher enough or not. Statistically, the larger the amount of learning samples and the higher performance the estimate method, the more accurate the prediction will be. It seems that EDAs does not care about how to take advantage of the independent behavior of each individual.

The basic ideas of EP and EDAs are quite different: EP uses the action of each individual, while EDAs use estimation with information included in population. It is interesting for us to see what results will be if these two kinds of optimization methodologies are connected together. On this point, this paper presents a hybrid evolutionary framework to make use of both the idea of EP and EDAs. Unlike previous EDAs that use probability density function(PDF), the estimation mechanism used in the proposed framework is the $k$-nearest neighbor estimation[16][17] which belongs to the same class of non-parametric estimation in machine learning as PDF estimation, and which performs better than PDF methods with relative small amount of training samples. In order to evaluate the performance of the proposed framework, both the Gaussian mutations which is with relative small jumps and Cauchy mutations which is with relative long jumps were used to have comparisons with their independent used algorithms that CEP and FEP respectively. Experimental results show that the guided CEP (GCEP) and the guided FEP (GFEP) outperform CEP and FEP significantly on many optimization problems respectively. This result conducts that the promotion out of machine learning is encouraging.

The rest of this paper is organized as follows. Section II gives a brief review of and primary analysis on EP's self-adaptive mutations, the basic ideas of EDAs, and nonparametric estimation in the field of machine learning. Section III introduces the $k$-nearest neighbor estimation and shows how to make prediction to guide the search of evolutionary programming. Thus a hybrid evolutionary framework was proposed. Section IV presents the experimental results on 23 benchmark functions to assess the performance of the proposed framework, where the impacts of the $k$-nearest neighbor estimation were also observed in detail according to the ratio used by EP. Section V gives the final conclusion and some remarks. 


\section{PReliminaries AND PRIMARY ANALYSis}

\section{A. Evolutionary Programming}

Optimization by Evolutionary Programming (EP) can be summarized into two major steps: 1) mutate the solutions in the current population; 2) select the next generation from the mutated and the current solutions. Each individual is taken as a pair of real-valued vectors $\left(\vec{x}_{i}, \vec{\eta}_{i}\right), \forall i \in$ $\{1, \cdots, \mu\}$, where $\vec{x}_{i}$ 's are objective vectors variables and $\vec{\eta}_{i}$ 's are standard deviations for mutations (also known as strategy parameters in self-adaptive evolutionary algorithms). For each current individual $\left(\vec{x}_{i}, \vec{\eta}_{i}\right)$, the update equations are

$$
\begin{gathered}
\vec{x}_{i}^{\prime}(j)=\vec{x}_{i}(j)+\vec{\eta}_{i}(j) N_{j}(0,1) \\
\vec{\eta}_{i}^{\prime}(j)=\vec{\eta}_{i}(j) \exp \left(\tau^{\prime} N(0,1)+\tau N_{j}(0,1)\right)
\end{gathered}
$$

where $\vec{x}_{i}(j), \vec{x}_{i}^{\prime}(j), \vec{\eta}_{i}(j)$, and $\vec{\eta}_{i}^{\prime}(j)$ denote the $j$-th component of the vectors $\vec{x}_{i}, \vec{x}_{i}^{\prime}, \vec{\eta}_{i}$ and $\vec{\eta}_{i}^{\prime}$, respectively. $N(0,1)$ denotes a normally distributed one-dimensional random number with mean zero and standard deviation one. $N_{j}(0,1)$ indicates that the random number is generated anew for each value of $j$. The factors $\tau$ and $\tau^{\prime}$ are commonly set to $(\sqrt{2 \sqrt{n}})^{-1}$ and $(\sqrt{2 n})^{-1}[11]$.

Based on above framework, Cauchy mutation and Lévy mutations are also studied. That is, they are introduced into Eq.(2) to substitute Gaussian mutation. Usually, EP using Gaussian mutations is called classical evolutionary programming(CEP), EP using Cauchy mutations is called fast evolutionary programming (FEP), and EP using Lévy mutations is called the adaptive LEP.

It is apparent, EP's mutations only focus on what probability distribution is more suitable for generating offsprings, and the self-adaption mechanism focus on how to enlarge or shrink the size of the search neighborhood.

\section{B. Estimation Distribution Algorithms}

According to [18], EDAs can be categorized into four classes. Algorithms belonging to the first class assume that the variance are independent of each other and the joint probability density function for $n$-dimensional vector $\vec{x}=\left(x_{1}, \cdots, x_{n}\right)$ can be factorized according to $p(\vec{x})=$ $\prod_{i=1}^{n} p\left(x_{i}\right)$; in class two, pairwise dependencies are considered; approaches of the third class take any multivariate interdependencies into account. EDAs of the last class use mixture distributions for conquering multimodal functions. Continuous optimization by EDAs can be summarized into the following framework[18].

1) Initialize a population of $N$ individuals randomly $\rightarrow D_{0}$

2) Select $\mathrm{Se} \leq N$ individuals from $D_{l-1}(l=1,2, \cdots)$ according to a selection method $\rightarrow D_{l-1}^{S e}$.

3) Estimate $n$-dimensional probability density function (PDF) based on $D_{l-1}^{S e} \rightarrow p_{l}(\vec{x})=p\left(\vec{x} \mid D_{l-1}^{S e}\right)$.

4) Sample $N$ new individuals from $p_{l}(\vec{x})$, from new population by partially of fully replacing the current population $\rightarrow D_{l}$.

5) Stop if some stoping criterion is reached, go to step 2 otherwise.
The basic ideas of EDAs are based on probability density function(PDF), that is the step 3 of above framework. In principle, PDF is corresponding to the Parzen-window[19] method in the field of machine learning. Therefore, EDAs have the same difficulties as those estimation methods of machine learning on condition when training samples are not much enough. Since results out of probability based estimations have risks unavoidably, it is not an appropriate manner that using EDAs without considering estimation errors. On this point of view, that EDAs together with nonestimation algorithms such as EP may be one of promising way.

\section{The Non-Parametric Estimation in Machine Learning}

The $k$-nearest neighbor[18] and Parzen windows[19] are belong to the same non-parametric estimation in machine learning. The basic ideas behind many of the methods of estimating an unknown probability density function are very simple. For non-parametric estimation, the most fundamental technique rely on

$$
\hat{p}_{N}(\vec{x}) \simeq \frac{k_{N} / N}{V_{N}}
$$

where $\hat{p}_{N}(\vec{x})$ denotes the estimated probability of vector $\vec{x}$ falling in the region $R_{N}$ with volume $V_{N}$ using $N$ samples, and $k_{N}$ means the number of samples falling in region $R_{N}$.

There are two common ways of obtaining sequences of regions that satisfies the convergent conditions[17]. One is to shrink an initial region by specifying the volume $V_{N}$ as some function of $N$, such as $V_{N}=1 / \sqrt{N}$. This is basically the Parzen-window method. The second is to specify $k_{N}$ as some function of $N$, such as $k_{N}=\sqrt{N}$. This is $k$-nearest neighbor estimation method.

The $k$-nearest neighbor rules, that the obvious extension of the $k$-nearest neighbor estimation, its estimate error $P$ have been proved within error bounds $P^{*} \leq P \leq 2 P^{*}[17]$, where $P^{*}$ denotes Bayes rate. The characteristic of the $k$-nearest neighbor rule different from Parzen-window method is that the former estimate posterior probability, while the later estimate the conditional density. Although non-parametric estimation requires sufficient samples, the $k$-nearest neighbor rule still possess better classification performance in the case of using deficient samples. On this point of view, the $k$ nearest neighbor estimation has the ability to guide EP's search in promising region.

\section{EVOlutionary Programming WITH $k$-NEAREST NEIGHBOR ESTIMATION}

\section{A. Promising Region and k-Nearest Neighbor Estimation}

A global minimization problem can be formalized as a pair $(S, f)$, where $S \subseteq R^{n}$ is a bounded set on $R^{n}$ and $f: S \mapsto R$ is an $n$-dimensional real-valued function. The problem is to find a point $x^{*} \in S$ such that $f\left(x^{*}\right)$ is a global minimum on $S$. More specifically, it is required to find an $x^{*} \in S$ such that

$$
\forall x \in S: f\left(x^{*}\right) \leq f(x)
$$


where $f$ does not need to be continuous but it must be bounded[6].

It is apparent, if the region where the global minima exist could be predicted by some mechanism, the space for search would be shrunk to a limited area. The more the predictive accuracy, the faster the convergence rate is, whereas the slower the the convergence rate is, since an missprediction may waste some computation resources and may make individuals fall into local minima. That is, there is an estimation risk of search with machine learning.

The $k$-nearest neighbor rule can partition the space into cells consisting all points closer to a given training point $x^{\prime}$ (an individual) than to any other training points(other individuals). All points in such a cell are thus lablled by the category of the training point - a so-called voroni tesselation of the space[17]. Nevertheless, according to the request of global optimization, the only concerned problem of optimization is where the global minimum exist in, although search space can be divided into several partitions with training samples. On this point of view, here we denote the cell which is predicted as the region where the global minima may exist possibly as $C_{P}$, and its representative point as $x_{P}$ which can be calculated as follows.

$$
x_{P}=\frac{1}{k} \sum_{i=1}^{k} x_{i}, \quad x_{i} \in S_{k} .
$$

As to the promising region that the cell $C_{P}$, the associated problem is how to generate offsprings in it. It is because the bounds of $C_{P}$ are usually irregular. If we want to generate offsprings within $C_{P}$ with the rule of voroni tesselation strictly, we will face the problem of constrained evolutionary optimization which is not wanted here. To deal with this problem, we can ignore the really shape of $C_{P}$, and replace it with a regular neighborhood of $x_{P}$ simply. Thus the method of generating offsprings in promising region can be as follows.

$$
x_{P}^{\prime}(j)=x_{P}(j)+\eta_{i}(j) U(-0.5,0.5),
$$

where $x_{P}^{\prime}$ denotes the new generated offspring in promising region, $U(-0.5,0.5)$ denotes an uniform random number bounded on $[-0.5,0.5]$, and $\eta_{i}(j)$ denotes the strategy parameter of $j$-th component of the $i$-th individual.

It is apparent, if the estimated promising region just covered the global minimum, the task of searching the global minimum could be achieved by generating many offsprings in $C_{P}$ greedily. However, prediction with machine learning are usually based on probability. In principle, the more the amount of training samples, the more larger the predictive accuracy, otherwise the more larger the estimation risk. Therefor in practice, we should limit the estimation works to a certain probability.

\section{B. The Framework of Introducing Estimation into Evolution- ary Programming}

In this section, we discuss EP algorithms for function optimization guided by the $k$-nearest neighbor estimation. The hybridisation framework of EP and the $k$-nearest neighbor estimation is as follows.

Step 1 Generate the initial population of $\mu$ individuals, and set $c=1$. Each individual is taken as a pair of realvalued vectors, $\left(X_{i}, \eta_{i}\right), \forall i \in\{1, \cdots, \mu\}$, where $X_{i}^{\prime} s$ are objective variables and $\eta_{i}^{\prime} s$ are strategy parameters in self-adaptive evolutionary algorithms.

Step 2 Evaluate the fitness score for each individual $\left(X_{i}, \eta_{i}\right), \forall i \in\{1, \cdots, \mu\}$, of the population based on the objective function, $f\left(X_{i}\right)$. Use $k$-nearest neighbor rule to get the reference vector $X_{P}$ by Eq.(3), where the $k$ means the number of "good" individuals, and can be set to 1 or $\sqrt{\mu}$ alternately.

Step 3 Each parent $\left(X_{i}, \eta_{i}\right), i=1, \cdots, \mu$, creates a temporary points $\left(X_{i}^{T}, \eta_{i}\right)$ in promising region with the probability $P_{k N N}$ by

$$
X_{i}^{T}(j)=X_{P}(j)+\eta_{i}(j) U(-0.5,0.5) .
$$

Otherwise,

$$
X_{i}^{T}(j)=X_{i}(j) .
$$

Step 4 For $j=1, \cdots, n$, each parent $\left(X_{i}^{T}, \eta_{i}\right), i=$ $1, \cdots, \mu$, creates a single offspring $\left(X_{i}^{\prime}, \eta_{i}^{\prime}\right)$ by

$$
\begin{gathered}
X_{i}^{\prime}(j)=X_{i}^{T}(j)+\eta_{i}(j) \psi_{j} \\
\eta_{i}^{\prime}(j)=\eta_{i}(j) \exp \left(\tau^{\prime} \psi+\tau \psi_{j}\right)
\end{gathered}
$$

where $X_{i}^{T}(j), X_{i}^{\prime}(j), \eta_{i}(j)$, and $\eta_{i}^{\prime}(j)$ denote the $j$-th component of the vectors $X_{i}^{T}, X_{i}^{\prime}, \eta_{i}$ and $\eta_{i}^{\prime}$, respectively. $\psi$ denotes an one-dimensional random number generated by a certain probability distribution. $\psi_{j}$ indicates that the random number is generated anew for each value of $j$. The factors $\tau$ and $\tau^{\prime}$ are commonly set to $(\sqrt{2 \sqrt{n}})^{-1}$ and $(\sqrt{2 n})^{-1}[6][11]$.

Step 5 Calculate the fitness of each offspring $\left(X_{i}^{\prime}, \eta_{i}^{\prime}\right), \forall i \in$ $\{1, \cdots, \mu\}$.

Step 6 Select the $\mu$ individuals out of the pair of $\left(X_{i}, \eta_{i}\right)$ and $\left(X_{i}^{\prime}, \eta_{i}^{\prime}\right), \forall i \in\{1, \cdots, \mu\}$, that have the better fitness score to be parents of the next generation.

Step 7 Stop if the halting criterion is satisfied; otherwise, $c=c+1$ and go to Step 2 .

It should be indicated, when $\psi$ denotes a Gaussian random number, the above framework becomes a guided CEP (GCEP); and when $\psi$ denotes a Cauchy random number, the above framework becomes a guided FEP (GFEP). Additionally, Gehlhaar and Fogel [8] showed that swapping the order of (7) and (8) may improve CEP's performance, while it seems have none of distinct improvement for the proposed framework.

\section{EXPERIMENTAL RESULTS}

In this section, we apply the proposed framework to function optimization problems given in Table I to view its performance, the strongpoint as well as the weakness. Functions $f_{1}-f_{13}$ are high-dimensional problems. Functions $f_{1}-$ $f_{5}$ are unimodal. Function $f_{6}$ is the step function, which has 
one minimum and is discontinuous. Function $f_{7}$ is a noisy quartic function. Functions $f_{8}-f_{13}$ are multimodal functions where the number of local minima increase exponentially with the problem dimensions. Functions $f_{14}-f_{23}$ are lowdimensional functions which have only a few local minima. In all experiments, we set population size $\mu=100$, the initial $\eta=3.0$. For CEP and FEP, the tournament size are both $q=10$ for selection (GCEP and GFEP do not use the tournament selection). The initial population was generated uniformly at random in the range as specified in descriptions of 23 benchmark functions.

According to different point of view, experiments are divided into two parts. Firstly, since estimation may make errors, it is necessary for us to view the impact of parameter $P_{k N N}$ on optimization performance. Then we can learn some experiences. Secondly, we should have a comparison between EP with estimation but without tournament technique(i.e., GCEP and GFEP) and EP with tournament technique but without estimation(i.e., CEP and FEP).

\section{A. Optimization Performance with Different Settings of Es- timation Parameters}

In order to view the impacts of parameter $P_{k N N}$ on optimization performance, here we set the probability used for estimation to $\{0,0.1,0.2,0.4,0.6,0.8,1\}$, where $P_{k N N}=0$ denotes EP is without estimation and tournament selection technique. Experimental results are summarized in Table II and Table III respectively. All results have been averaged over 50 runs. Based on these experimental results, we have analysis and experiences as follows.

- Without estimation and tournament selection technique, i.e., the case $P_{k N N}=0$, EP's performance (both in using Gaussian and Cauchy mutations) degraded sharply on all high-dimensional problems $\left(f_{1}-f_{13}\right)$. This results shows that the tournament selection technique plays an important role but is not the only way, since introducing estimation into EP's search cycle can also improve EP's performance evidently, however have different results with different setting of parameter $P_{k N N}$.

- It is also detrimental to improving EP's performance that piling too many expectations on the capability of estimation. The results of EP with estimation in the case of $P_{k N N}=1$ showed the trouble clearly. The matter is reasonable, since it is unavoidable that estimation may make errors. In this case, optimization performance is also decided by the ability of escaping local minima of mutations.

- For GCEP which uses Gaussian mutations, it seems that it with $P_{k N N}=0.1$ can make relative better results than with other settings in the mass. While for GFEP which uses Cauchy mutations, it seems that it with $P_{k N N}=0.2$ can make relative better results than with other settings in the mass. This result shows that the setting of $P_{k N N}$ is better with relative small value.

\section{B. Comparison with CEP and FEP}

CEP and FEP use tournament selection technique, while GCEP and GFEP use $k$-nearest neighbor estimation. It is necessary to having comparisons between GCEP and CEP, as well as between GFEP and FEP to show how the power estimation is. For the GCEP and the GFEP, we set $P_{k N N}=$ 0.1 and $P_{k N N}=0.2$ respectively according to the cognitive experiences about the relation between evolution and estimation. That is, Cauchy mutation can escape local minima much faster than Gaussian mutation, when the $k$-nearest neighbor estimation makes incorrect predictions. Experimental results are summarized in Table IV. All results have been averaged over 50 runs. It is apparent, GCEP and GFEP performs better than CEP and FEP significantly on many benchmark functions respectively.

In order to view what profits that EP can obtain from $k$ nearest neighbor estimation, and how importance that long jumps play in evolution, we take out some operations from GFEP to do experiments. The one, we do not use estimation and tournament selection technique, i.e., we run the proposed framework without Eq.(5). Conveniently, we call the framework with this setting the bare-FEP. The other, we do not use mutation and tournament selection technique, i.e., we run the proposed framework without Eq.(7). Conveniently, we call the framework with this setting the bare-estimation. Fig. 1, Fig.2, and Fig. 3 showed the averaged optimization curves of these two settings and GFEP with $P_{k N N}=0.2$ on unimodal functions $f_{1}$ and $f_{3}$, and multimodal function $f_{8}$. It is clear, estimation is good at making prediction when the global minimum is easy being learned, while Cauchy mutation is good at helping individuals escaping local minima when estimation losses some predictive accuracy. The hybridiastion of EP with Cauchy mutation and the $k$-nearest neighbor estimation seems that it can produce a promising solution.

\section{Conclusions}

Evolutionary programming (EP) is one of the most important optimization methodology in the field of evolutionary computation, while Estimation of Distribution Algorithms (EDAs) are another approach method of population based search algorithms that generate new population from the estimated distribution based on current promising solutions. This paper presents a hybrid evolutionary framework to make use of both the idea of EP and EDAs. Unlike previous EDAs that use probability density function(PDF), the estimation mechanism used in the proposed framework is the $k$-nearest neighbor estimation which had been proved being better than PDF methods with relative small amount of training samples in the field of machine learning. Experimental study shows that the idea of EP and EDAs can be connected to make reinforce each other: estimation can guide EP's search in promising region, while EP's long jumps can help those poorguided individuals escaping local minima efficiently. Our primary results showed, although the proposed framework is simple yet and not so perfect, it is a promising way to make benefits using different ideas of evolution together. 
TABLE I

THE 23 BENCHMARK FUNCTIONS USED FOR EXPERIMENTS, WHERE $n$ IS THE DIMENSION OF THE FUNCTION, $f_{\text {min }}$ IS THE MINIMUM VALUE OF THE FUNCTION, AND $S \subseteq R^{n}$. A DETAILED DESCRIPTION OF ALL FUNCTIONS SEE [6].

\begin{tabular}{|c|c|c|c|}
\hline Test Functions & $n$ & $S$ & $f_{\min }$ \\
\hline$f_{1}(x)=\sum_{i=1}^{30} x_{i}^{2}$ & 30 & {$[-100,100]^{n}$} & 0 \\
\hline$f_{2}(x)=\sum_{i=1}^{30}\left|x_{i}\right|+\prod_{i=1}^{30}\left|x_{i}\right|$ & 30 & {$[-10,10]^{n}$} & 0 \\
\hline$f_{3}(x)=\sum_{i=1}^{30}\left(\sum_{j=1}^{i} x_{j}\right)^{2}$ & 30 & {$[-100,100]^{n}$} & 0 \\
\hline$f_{4}(x)=\max _{i}\left\{\left|x_{i}\right|, \quad 1 \leq i \leq n\right\}$ & 30 & {$[-100,100]^{n}$} & 0 \\
\hline$f_{5}(x)=\sum_{\substack{i=1 \\
30}}^{29}\left[100\left(x_{i+1}-x_{i}^{2}\right)^{2}+\left(x_{i}-1\right)^{2}\right]$ & 30 & {$[-30,30]^{n}$} & 0 \\
\hline$f_{6}(x)=\sum_{i}^{\infty}\left(\left\lfloor x_{i}+0.5\right\rfloor\right)^{2}$ & 30 & {$[-100,100]^{n}$} & 0 \\
\hline$f_{7}(x)=\sum_{i}^{30} i x_{i}^{4}+$ random $[0,1)$ & 30 & {$[-1.28,1.28]^{n}$} & 0 \\
\hline$f_{8}(x)=-\sum_{i}^{30}\left(x_{i} \sin \left(\sqrt{\left|x_{i}\right|}\right)\right)$ & 30 & {$[-500,500]^{n}$} & -12569.5 \\
\hline$f_{9}(x)=\sum_{i}\left[x_{i}^{2}-10 \cos \left(2 \pi x_{i}\right)+10\right]$ & 30 & {$[-5.12,5.12]^{n}$} & 0 \\
\hline 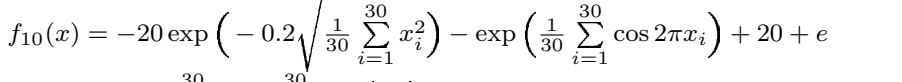 & 30 & {$[-32,32]^{n}$} & 0 \\
\hline$f_{11}(x)=\frac{1}{4000} \sum_{i=1}^{30} x_{i}^{2}-\prod_{i=1}^{30} \cos \left(\frac{x_{i}}{\sqrt{i}}\right)+1$ & 30 & {$[-600,600]^{n}$} & 0 \\
\hline $\begin{aligned} f_{12}(x)= & \frac{\pi}{30}\left\{10 \sin ^{2}\left(\pi y_{1}\right)+\sum_{i=1}^{29}\left(y_{i}-1\right)^{2}\left[1+10 \sin ^{2}\left(\pi y_{i+1}\right)\right]+\left(y_{n}-1\right)^{2}\right\} \\
& +\sum_{i=1}^{30} u\left(x_{i}, 10,100,4\right), y_{i}=1+\frac{1}{4}\left(x_{i}+1\right)\end{aligned}$ & 30 & {$[-50,50]^{n}$} & 0 \\
\hline $\begin{aligned} f_{13}(x)= & 0.1\left\{10 \sin ^{2}\left(\pi 3 x_{1}\right)+\sum_{i=1}^{29}\left(x_{i}-1\right)^{2}\left[1+\sin ^{2}\left(3 \pi x_{i+1}\right)\right]+\left(x_{n}-1\right)^{2}[1+\right. \\
& \left.\left.\sin ^{2}\left(2 \pi x_{30}\right)\right]\right\}+\sum_{i=1}^{30} u\left(x_{i}, 5,100,4\right)\end{aligned}$ & 30 & {$[-50,50]^{n}$} & 0 \\
\hline$f_{14}(x)=\left[\frac{1}{500}+\sum_{j=1}^{25} \frac{1}{j+{ }_{i=1}^{2}\left(x_{i}-a_{i j}\right)^{6}}\right]^{-1}$ & 2 & {$[-65.536,65.536]^{n}$} & 0.998 \\
\hline$f_{15}(x)=\sum_{i=1}^{11}\left[a_{i}-\frac{x_{1}\left(b_{i}^{2}+b_{i} x_{2}\right)}{b_{i}^{2}+b_{i} x_{3}+x_{4}}\right]^{2}$ & 4 & {$[-5,5]^{n}$} & 0.0003075 \\
\hline$f_{16}(x)=4 x_{1}^{2}-2.1 x_{1}^{4}+\frac{1}{3} x_{1}^{6}+x_{1} x_{2}-4 x_{2}^{2}+4 x_{2}^{4}$ & 2 & {$[-5,5]^{n}$} & -1.0316285 \\
\hline$f_{17}(x)=\left(x_{2}-\frac{5.1}{4 \pi^{2}} x_{1}^{2}+\frac{5}{\pi} x_{1}-6\right)^{2}+10\left(1-\frac{1}{8 \pi}\right) \cos x_{1}+10$ & 2 & {$[-5,10] \times[0,15]$} & 0.398 \\
\hline $\begin{aligned} & f_{18}(x)= {\left[1+\left(x_{1}+x_{2}+1\right)^{2}\left(19-14 x_{1}+3 x_{1}^{2}-14 x_{2}+6 x_{1} x_{2}+3 x_{2}^{2}\right)\right] } \\
& \times\left[30+\left(2 x_{1}-3 x_{2}\right)^{2} \times\left(18-32 x_{1}+12 x_{1}^{2}+48 x_{2}-36 x_{1} x_{2}+27 x_{2}^{2}\right)\right]\end{aligned}$ & 2 & {$[-2,2]^{n}$} & 3 \\
\hline$f_{19}(x)=-\sum_{i=1}^{4} c_{i} \exp \left[-\sum_{j=1}^{4} a_{i j}\left(x_{j}-p_{i j}\right)^{2}\right]$ & 4 & {$[0,1]^{n}$} & -3.86 \\
\hline$f_{20}(x)=-\sum_{i=1}^{4} c_{i} \exp \left[-\sum_{j=1}^{6} a_{i j}\left(x_{j}-p_{i j}\right)^{2}\right]$ & 6 & {$[0,1]^{n}$} & -3.32 \\
\hline$f_{21}(x)=-\sum_{i=1}^{5}\left[\left(x-a_{i}\right)\left(x-a_{i}\right)^{T}+c_{i}\right]^{-1}$ & 4 & {$[0,10]^{n}$} & -10 \\
\hline$f_{22}(x)=-\sum_{i=1}^{7}\left[\left(x-a_{i}\right)\left(x-a_{i}\right)^{T}+c_{i}\right]^{-1}$ & 4 & {$[0,10]^{n}$} & -10 \\
\hline$f_{23}(x)=-\sum_{i=1}^{10}\left[\left(x-a_{i}\right)\left(x-a_{i}\right)^{T}+c_{i}\right]^{-1}$ & 4 & {$[0,10]^{n}$} & -10 \\
\hline
\end{tabular}


TABLE II

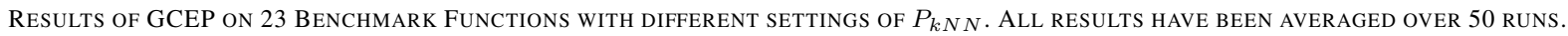

\begin{tabular}{|c|c|c|c|c|c|c|c|c|}
\hline Functions & Generations & $P_{k N N}=0$ & $P_{k N N}=0.1$ & $P_{k N N}=0.2$ & $P_{k N N}=0.4$ & $P_{k N N}=0.6$ & $P_{k N N}=0.8$ & $P_{k N N}=1$ \\
\hline$f_{1}$ & 1500 & 383.89 & $8.92 e-5$ & $7.63 e-5$ & $4.91 e-5$ & $2.36 e-5$ & $6.04 e-5$ & $4.61 e-5$ \\
\hline$f_{2}$ & 2000 & 35.40 & $2.95 e-5$ & $3.38 e-2$ & $1.37 e-2$ & $3.13 e-2$ & $2.23 e-2$ & $2.16 e^{-2}$ \\
\hline$f_{3}$ & 5000 & $2.97 e+3$ & 1.08 & 0.1740 & $7.50 e-3$ & $7.8 e-3$ & $2.10 e-3$ & $2.10 e-3$ \\
\hline$f_{4}$ & 5000 & 18.61 & $2.71 e-2$ & $6.30 e-3$ & $1.7 e-3$ & $1.0 e-3$ & $7.84 e-4$ & $5.47 e-4$ \\
\hline$f_{5}$ & 20000 & 36.34 & 27.54 & 20.47 & 32.18 & 28.89 & 16.61 & 22.70 \\
\hline$f_{6}$ & 1500 & 436.1 & 0 & 0 & 0 & 0 & 0 & 0 \\
\hline$f_{7}$ & 3000 & 0.147 & $8.60 e-3$ & $5.7 e-3$ & $4.3 e-3$ & $3.9 e-3$ & $5.1 e-3$ & $3.5 e-3$ \\
\hline$f_{8}$ & 9000 & -8379.3 & -11104.8 & -10984 & -11043 & -11065 & -11138 & -11290 \\
\hline$f_{9}$ & 5000 & 124.57 & 21.71 & 20.96 & 17.84 & 21.75 & 22.79 & 26.48 \\
\hline$f_{10}$ & 1500 & 10.02 & 0.17 & $6.02 e-2$ & 0.10 & 0.11 & 0.36 & 0.30 \\
\hline$f_{11}$ & 2000 & 4.0 & $1.01 e-2$ & $1.55 e-2$ & $6.9 e-3$ & $9.3 e-3$ & $9.4 e-3$ & $9.6 e-3$ \\
\hline$f_{12}$ & 1500 & 18.71 & 5.43 & 2.34 & 5.70 & 7.12 & 5.87 & 6.59 \\
\hline$f_{13}$ & 1500 & 108.88 & $4.20 e-3$ & $5.32 e-5$ & $1.1 e-3$ & $1.74 e-5$ & $3.51 e-5$ & $1.1 e-3$ \\
\hline$f_{14}$ & 100 & 1.000 & 0.998 & 0.998 & 0.998 & 0.998 & 0.998 & 0.998 \\
\hline$f_{15}$ & 4000 & $3.33 e-4$ & $3.99 e-4$ & $3.075 e-4$ & $5.92 e-4$ & $4.91 e-4$ & $3.99 e-4$ & $3.99 e 10^{-4}$ \\
\hline$f_{16}$ & 100 & -1.0316 & -1.0316 & -1.0316 & -1.0316 & -1.0316 & -1.0316 & -1.0316 \\
\hline$f_{17}$ & 100 & 0.3979 & 0.3979 & 0.3979 & 0.3979 & 0.3979 & 0.3979 & 0.3979 \\
\hline$f_{18}$ & 100 & 3.0001 & 3.0 & 3.0 & 3.0 & 3.0 & 3.0 & 3.0 \\
\hline$f_{19}$ & 100 & -3.8627 & -3.8628 & -3.8628 & -3.8628 & -3.8628 & -3.8628 & -3.8628 \\
\hline$f_{20}$ & 200 & 3.3175 & -3.3220 & -3.3220 & -3.3101 & -3.31 & -3.3101 & -3.2863 \\
\hline$f_{21}$ & 100 & -8.9288 & -10.145 & -8.6025 & -7.0936 & -7.0943 & -6.0746 & -6.3473 \\
\hline$f_{22}$ & 100 & -9.8573 & -10.042 & -9.3397 & -8.8081 & -9.3397 & -7.2137 & -7.2137 \\
\hline$f_{23}$ & 100 & -9.6768 & -10.535 & -10.5360 & -9.4545 & -9.9955 & -8.9139 & -9.4548 \\
\hline
\end{tabular}

TABLE III

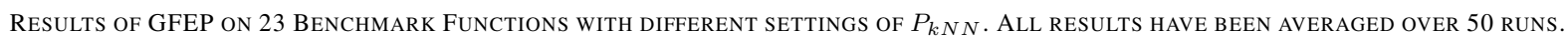

\begin{tabular}{|c|c|c|c|c|c|c|c|c|}
\hline Functions & Generations & $P_{k N N}=0$ & $P_{k N N}=0.1$ & $P_{k N N}=0.2$ & $P_{k N N}=0.4$ & $P_{k N N}=0.6$ & $P_{k N N}=0.8$ & $P_{k N N}=1$ \\
\hline$f_{1}$ & 1500 & 565.05 & $1.38 e-7$ & $1.85 e-8$ & $1.42 e-8$ & $1.50 e-8$ & $1.43 e-8$ & $1.66 e-8$ \\
\hline$f_{2}$ & 2000 & 20.10 & $6.35 e-4$ & $5.22 e-4$ & $5.16 e-4$ & $5.14 e-4$ & $5.29 e-4$ & $5.29 e-4$ \\
\hline$f_{3}$ & 5000 & $3.97 e+3$ & 3.2 & 0.39 & $3.47 e-2$ & $1.9 e-3$ & $9.43 e-4$ & $1.88 e-4$ \\
\hline$f_{4}$ & 5000 & 17.06 & $2.3 e-2$ & $4.2 e-3$ & $6.17 e-4$ & $2.16 e-4$ & $8.08 e-5$ & $6.72 e-5$ \\
\hline$f_{5}$ & 20000 & 16.34 & 29.40 & 13.40 & 8.38 & 8.78 & 8.38 & 1.68 \\
\hline$f_{6}$ & 1500 & 691.20 & 0 & 0 & 0 & 0 & 0 & 0 \\
\hline$f_{7}$ & 3000 & 0.21 & $1.17 e-2$ & $1.18 e-2$ & $8.9 e-3$ & $7.0 e-3$ & $7.1 e-3$ & $7.7 e-3$ \\
\hline$f_{8}$ & 9000 & -10285 & -12546.0 & -12569.5 & -12569.5 & -12569.5 & -12556.6 & -12534 \\
\hline$f_{9}$ & 5000 & 76.95 & 0.80 & $2.59 e-6$ & $9.95 e-2$ & $2.22 e-6$ & $9.95 e-2$ & $9.95 e-2$ \\
\hline$f_{10}$ & 1500 & 9.94 & $6.58 e-4$ & $1.19 e-4$ & $8.96 e-5$ & $8.69 e-5$ & $9.02 e-5$ & $9.27 e-5$ \\
\hline$f_{11}$ & 2000 & 3.5147 & $1.97 e-2$ & $1.60 e-2$ & $1.45 e-2$ & $2.29 e-2$ & $2.60 e-2$ & $1.84 e-2$ \\
\hline$f_{12}$ & 1500 & 23.30 & $4.60 e-6$ & $6.21 e-9$ & $3.22 e-10$ & $1.79 e-10$ & $1.64 e-10$ & $1.62 e-10$ \\
\hline$f_{13}$ & 1500 & $9.97 e+3$ & $2.00 e-6$ & $1.26 e-8$ & $3.78 e-9$ & $2.54 e-9$ & $2.60 e-9$ & $2.75 e-9$ \\
\hline$f_{14}$ & 100 & 0.998 & 0.998 & 0.998 & 0.998 & 0.998 & 0.998 & 0.998 \\
\hline$f_{15}$ & 4000 & $3.61 e-4$ & $3.22 e-4$ & $4.11 e-4$ & $3.518 e-4$ & $3.075 e-4$ & $3.991 e-4$ & $6.193 e-4$ \\
\hline$f_{16}$ & 100 & -1.0316 & -1.0316 & -1.0316 & -1.0316 & -1.0316 & -1.0316 & -1.0316 \\
\hline$f_{17}$ & 100 & 0.3979 & 0.3979 & 0.3979 & 0.3979 & 0.3979 & 0.3979 & 0.3979 \\
\hline$f_{18}$ & 100 & 3.0041 & 3.0 & 3.0 & 3.0 & 3.0 & 3.0 & 3.0 \\
\hline$f_{19}$ & 100 & -3.8625 & -3.8628 & -3.8628 & -3.8628 & -3.8628 & -3.8628 & -3.8628 \\
\hline$f_{20}$ & 200 & -3.3113 & -3.3218 & -3.2982 & -3.2982 & -3.2625 & -3.2625 & -3.2863 \\
\hline$f_{21}$ & 100 & -7.9771 & -8.53 & -8.1137 & -7.3670 & -8.1140 & -7.6042 & -6.5846 \\
\hline$f_{22}$ & 100 & -8.7775 & -8.81 & -9.3284 & -8.2768 & -6.4096 & -8.7956 & -9.2035 \\
\hline$f_{23}$ & 100 & -8.9836 & -10.00 & -9.9261 & -5.9515 & -7.7031 & -7.4445 & -6.8917 \\
\hline
\end{tabular}


TABLE IV

FINAL SOLUTION OF CEP, FEP, GCEP, AND GFEP ON 23 BENCHMARK FUNCTIONS, WHERE THE VALUE OF $t$ WITH 49 DEGREES OF FREEDOM IS SIGNIFICANT AT $\alpha=0.05$ BY A TWO-TAILED TEST. ALL RESULTS HAVE BEEN AVERAGED OVER 50 RUNS.

\begin{tabular}{|c|c|c|c|c|c|c|c|}
\hline \multirow{2}{*}{$\begin{array}{l}\text { Test } \\
\text { Functions }\end{array}$} & \multicolumn{3}{|c|}{ GCEP } & \multicolumn{3}{|c|}{ CEP } & \multirow{2}{*}{$\begin{array}{c}\text { GCEP-CEP } \\
t \text {-test }\end{array}$} \\
\hline & Generations & Mean Best & Std. Dev & Generations & Mean Best & Std. Dev & \\
\hline$f_{1}$ & 1500 & $8.92 e-5$ & $4.86 e-5$ & 1500 & $2.2 e-4$ & $5.9 e-4$ & -1.5623 \\
\hline$f_{2}$ & 2000 & $2.95 e-5$ & $3.35 e-5$ & 2000 & $2.6 e-3$ & $1.7 e-4$ & $-104.9 \dagger$ \\
\hline$f_{3}$ & 5000 & 1.08 & 1.00 & 5000 & $5.0 e-2$ & $6.6 e-2$ & $7.2674 \dagger$ \\
\hline$f_{4}$ & 5000 & $2.71 e-2$ & $1.17 e-2$ & 5000 & 2.0 & 1.2 & $-11.625 \dagger$ \\
\hline$f_{5}$ & 20000 & 27.54 & 17.29 & 20000 & 6.17 & 5.87 & $8.2757 \dagger$ \\
\hline$f_{6}$ & 1500 & 0 & 0 & 1500 & 577.76 & 1125.76 & $-3.629 \dagger$ \\
\hline$f_{7}$ & 3000 & $8.60 e-3$ & $3.4 e-3$ & 3000 & $1.8 e-2$ & $6.4 e-3$ & $-9.1717 \dagger$ \\
\hline$f_{8}$ & 9000 & -11104.8 & 334.31 & 9000 & -7917.1 & 634.5 & -31.433 \\
\hline$f_{9}$ & 5000 & 21.71 & 7.65 & 5000 & 89.0 & 23.1 & $-19.554 \dagger$ \\
\hline$f_{10}$ & 1500 & 0.17 & $4.81 e-1$ & 1500 & 9.2 & 2.8 & $-22.475 \dagger$ \\
\hline$f_{11}$ & 2000 & $1.01 e-2$ & $1.66 e-2$ & 2000 & $8.6 e-2$ & 0.12 & $-4.4303 \dagger$ \\
\hline$f_{12}$ & 1500 & 5.43 & 2.54 & 1500 & 1.76 & 2.4 & $7.4262 \dagger$ \\
\hline$f_{13}$ & 1500 & $4.20 e-3$ & $1.21 e-2$ & 1500 & 1.4 & 3.7 & $-2.6675 \dagger$ \\
\hline$f_{14}$ & 100 & 0.998 & $1.37 e-13$ & 100 & 1.66 & 1.19 & $-3.9337 \dagger$ \\
\hline$f_{15}$ & 4000 & $3.99 e-4$ & $2.90 e-4$ & 4000 & $4.7 e-4$ & $3.0 e-4$ & -1.2032 \\
\hline$f_{16}$ & 100 & -1.03 & $8.65 e-9$ & 100 & -1.03 & $4.9 e-7$ & 0 \\
\hline$f_{17}$ & 100 & 0.398 & $2.75 e-9$ & 100 & 0.398 & $1.5 e-7$ & 0 \\
\hline$f_{18}$ & 100 & 3.0 & $9.07 e-8$ & 100 & 3.0 & 0 & 0 \\
\hline$f_{19}$ & 100 & -3.8628 & $9.88 e-7$ & 100 & -3.86 & $1.4 e-2$ & $-1.4142 \dagger$ \\
\hline$f_{20}$ & 200 & -3.3220 & $1.42 e-6$ & 200 & -3.28 & $5.8 e-2$ & $-5.1204 \dagger$ \\
\hline$f_{21}$ & 100 & -10.145 & $1.38 e-2$ & 100 & -6.86 & 2.67 & $-8.6997 \dagger$ \\
\hline$f_{22}$ & 100 & -10.042 & 1.14 & 100 & -8.27 & 2.95 & $-3.9621 \dagger$ \\
\hline$f_{23}$ & 100 & -10.535 & $1.50 e-3$ & 100 & -9.1 & 2.92 & $-3.4750 \dagger$ \\
\hline Test & & GFEP & & & FEP & & GFEP-FEP \\
\hline Functions & Generations & Mean Best & Std. Dev & Generations & Mean Best & Std. Dev & $t$-test \\
\hline$f_{1}$ & 1500 & $1.85 e-8$ & $2.00 e-9$ & 1500 & $5.7 e-4$ & $1.3 e-4$ & $-31.003 \dagger$ \\
\hline$f_{2}$ & 2000 & $5.22 e-4$ & $5.59 e-5$ & 2000 & $8.1 e-3$ & $7.7 e-4$ & $-69.408 \dagger$ \\
\hline$f_{3}$ & 5000 & 0.39 & 0.25 & 5000 & $1.6 e-2$ & $1.4 e-2$ & $10.562 \dagger$ \\
\hline$f_{4}$ & 5000 & $4.2 e-3$ & $1.8 e-3$ & 5000 & 0.3 & 0.5 & $-4.1832 \dagger$ \\
\hline$f_{5}$ & 20000 & 13.40 & 24.33 & 20000 & 5.06 & 5.87 & $2.3563 \dagger$ \\
\hline$f_{6}$ & 1500 & 0 & 0 & 1500 & 0 & 0 & 0 \\
\hline$f_{7}$ & 3000 & $1.18 e-2$ & $3.3 e-3$ & 3000 & $7.6 e-3$ & $2.6 e-3$ & $7.0691 \dagger$ \\
\hline$f_{8}$ & 9000 & -12569.5 & $1.0 e-3$ & 9000 & -12554.5 & 52.6 & -2.0165 \\
\hline$f_{9}$ & 5000 & $2.59 e-6$ & $1.22 e-6$ & 5000 & $4.6 e-2$ & $1.2 e-2$ & $-27.104 \dagger$ \\
\hline$f_{10}$ & 1500 & $1.19 e-4$ & $6.32 e-5$ & 1500 & $1.8 e-2$ & $2.1 e-3$ & $-60.181 \dagger$ \\
\hline$f_{11}$ & 2000 & $1.60 e-2$ & $1.81 e-2$ & 2000 & $1.6 e-2$ & $2.2 e-2$ & 0 \\
\hline$f_{12}$ & 1500 & $6.21 e-9$ & $7.25 e-9$ & 1500 & $9.2 e-6$ & $3.6 e-6$ & $-18.058 \dagger$ \\
\hline$f_{13}$ & 1500 & $1.26 e-8$ & $5.37 e-9$ & 1500 & $1.6 e-4$ & $7.3 e-5$ & $-15.497 \dagger$ \\
\hline$f_{14}$ & 100 & 0.998 & $1.66 e-16$ & 100 & 1.22 & 0.56 & $-2.8032 \dagger$ \\
\hline$f_{15}$ & 4000 & $4.11 e-4$ & $3.24 e-4$ & 4000 & $5 e-4$ & $3.2 e-6$ & -1.9423 \\
\hline$f_{16}$ & 100 & -1.0316 & $8.06 e-11$ & 100 & -1.031 & $4.9 e-7$ & $-8658.5 \dagger$ \\
\hline$f_{17}$ & 100 & 0.398 & $2.20 e-11$ & 100 & 0.398 & $1.5 e-7$ & 0 \\
\hline$f_{18}$ & 100 & 3.0 & $5.39 e-9$ & 100 & 3.02 & 0.11 & -1.2856 \\
\hline$f_{19}$ & 100 & -3.8628 & $4.94 e-9$ & 100 & -3.86 & $1.4 e-5$ & $-1414.2 \dagger$ \\
\hline$f_{20}$ & 200 & -3.2982 & $5.01 e-2$ & 200 & -3.27 & $5.9 e-2$ & $-2.5762 \dagger$ \\
\hline$f_{21}$ & 100 & -8.1137 & 2.63 & 100 & -5.52 & 1.59 & $-5.9677 \dagger$ \\
\hline$f_{22}$ & 100 & -9.3284 & 2.24 & 100 & -5.52 & 2.12 & $-8.7316 \dagger$ \\
\hline$f_{23}$ & 100 & -9.9261 & 1.70 & 100 & -6.57 & 3.14 & $-6.6462 \dagger$ \\
\hline
\end{tabular}




\section{REFERENCES}

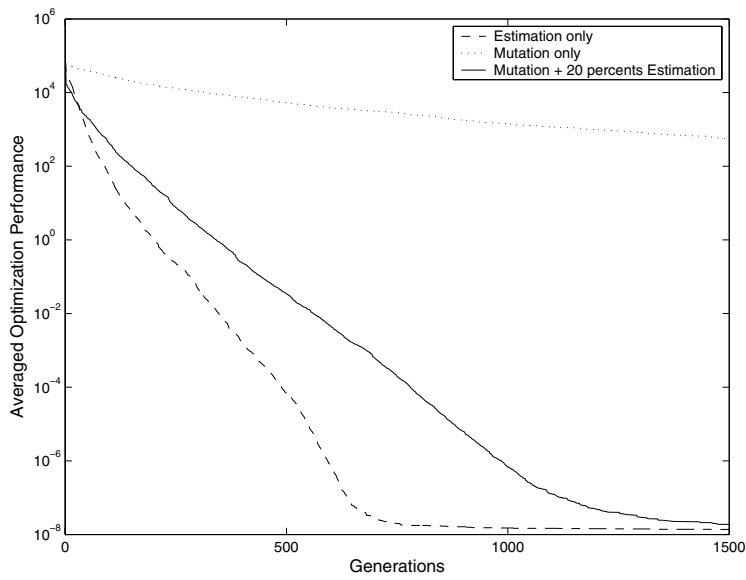

Fig. 1. Averaged optimization curves of the bare-FEP, bare-estimation, and GFEP with $P_{k N N}=0.2$ on unimodal function $f_{1}$.

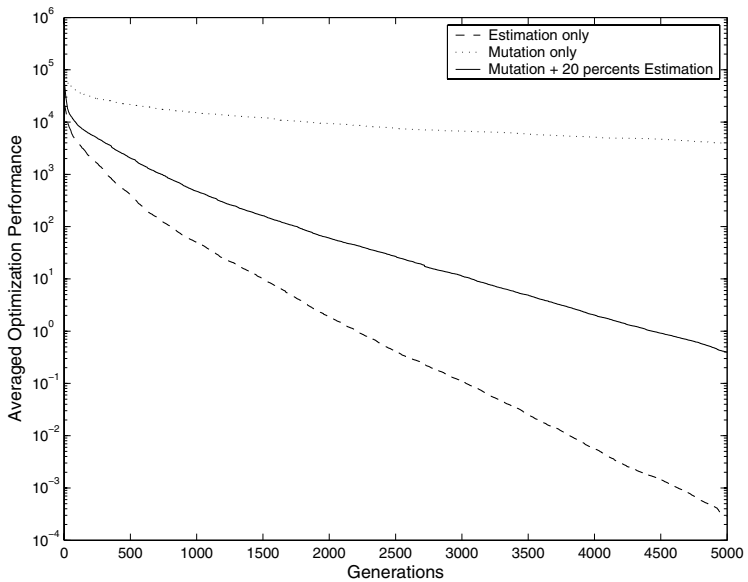

Fig. 2. Averaged optimization curves of the bare-FEP, bare-estimation, and GFEP with $P_{k N N}=0.2$ on unimodal function $f_{3}$.

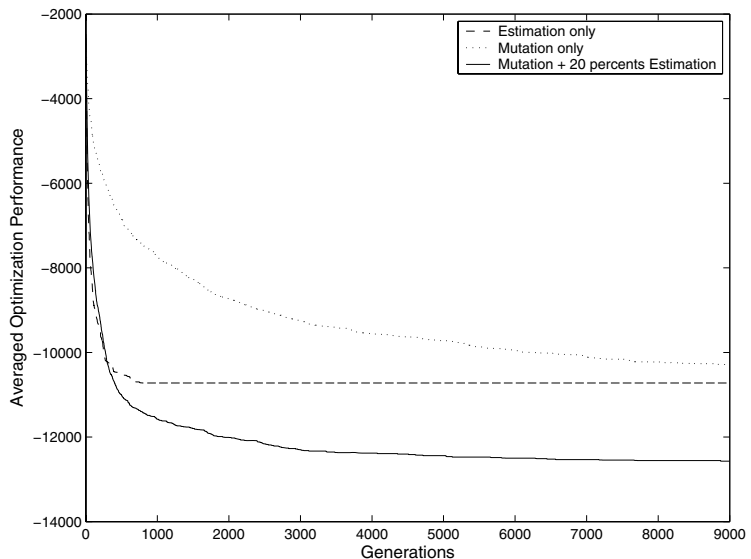

Fig. 3. Averaged optimization curves of the bare-FEP, bare-estimation, and GFEP with $P_{k N N}=0.2$ on multimodal function $f_{8}$.
[1] L. J. Fogel, A. J. Owens, and M. J. Walsh, Artificial Intelligence Through Simulated Evolution. New York: Wiley, 1966.

[2] D. B. Fogel, System Identification Through Simulated Evolution: A Machine Learning Approach to Modeling. Needham Heights, MA: Ginn, 1991.

[3] D. B. Fogel, "Evolving artificial intelligence," $\mathrm{Ph}$.D. dissertation, Univ. of California, San Diego, CA, 1992.

[4] D. B. Fogel, "Applying evolutionary programming to selected traveling salesman problems," Cybern. Syst., Vol. 24, pp. 27-36, 1993.

[5] B.Li, J.Lin, X.Yao, "A novel evolutionary algorithm for determining unified creep damage constitutive equations," International Journal of Mechanical Sciences, Vol. 44, pp. 987-1002, 2002.

[6] X. Yao, Y. Liu, and G. Lin, "Evolutionary programming made faster," IEEE Transactions on Evolutionary Computation, Vol.3, No.2, pp. 82102, July 1999

[7] C. Y. Lee and X. Yao, "Evolutionary programming using the mutations based on Lévy probability distribution," IEEE Transactions on Evolutionary Computation, Vol. 8, no. 5, October 2004, pp. 456-470.

[8] D. K. Gehlharr and D. B. Fogel, "Tuning evolutionary programming for conformationally flexible molecular docking," in Evolutionary Programming V: Proc. of the Fifth Annual Conference on Evolutionary Programming, L. J. Fogel, P. J. Angeline, and T. Bäck, EDs. Cambridge, MA: MIT Press, 1996, pp. 419-429.

[9] H.-P. Schwefel, Evolution and Optimum Seeking. New York: Wiley, 1995.

[10] D. B. Fogel, Evolutionary Computation: Tward a New Philosophy of Machine Intelligence. Piscataway, NJ: IEEE Press, 1995.

[11] T. Bäck and H. -P. Schwefel, "An overview of evolutionary algorithms for parameter optimization," Evol. Comput., Vol.1, No.1, pp. 1-23, 1993.

[12] M. Gallagher, M. Frean, and T. Downs, "Real-valued evolutionary optimization using a flexible probability density estimator," in Proc. Genetic and Evolutionary Computation Conf. (GECCO'99), Vol.1, 1999, pp. 840-846.

[13] M. Pelikan and D. E. Goldberg, "Genetic algorithms, clustering, and the breaking of symmetry," in Proc. Parallel Problem Solving from Nature - PPSN VI, Paris, France, 2000, pp. 385-394.

[14] P. Larrañaga and J. A. Lozano, Eds., Estimation of Distribution Algorithms: A New Tool for Evolutionary Computation. Norwell, MA: Kluwer, 2001, ch. 4, pp. 101-127.

[15] P. A. N. Bosnian and D. Thierens, "Advancing continuous IDEA's with mixture distributions and factorization selection metrics," in Proc. Optimization by Building and Using Probabilistic Models (OBUPM) Workshop at the Genetic and Ecolutionary Computation Conf. (GECCO2001), M. Pelikan and K. Sastry, Eds., San Francisco, CA, 2001, pp. 208-212.

[16] Thomas M. Cover and Peter E. Hart, "Nearest neighbor pattern classification," IEEE Trans. on Information Theory, IT-13(1): 21-27, 1967.

[17] Richard O. Duda, Peter E. Hart, and David G. Stork: Pattern Classification, Second Edition, New York: John Wily \& Sons, 2001.

[18] Q. Lu and X. Yao, "Clustering and Learning Gaussian Distribution for Continuous Optimization," IEEE Trans. on System, Man, and Cybernetics, Part C: Applications and Reviews, Vol. 35, No. 2, May 2005, pp.195-204

[19] Emanuel Parzen. On estimation of a probability density function and mode. Annals of Mathematical Statistics, 33(3):1065-1076,1962. 